Review Article

\title{
Efficacy and Safety of Stem Cell Combination Therapy for Osteonecrosis of the Femoral Head: A Systematic Review and Meta-Analysis
}

\author{
Junqiao Li $(\mathbb{D}$, Peng Su, Jian Li, Gang Chen, and Yan Xiong \\ Department of Orthopaedic Surgery, Orthopedic Research Institute, West China Hospital, Sichuan University, 37 Guoxue Lane, \\ Wuhou District, Chengdu, China \\ Correspondence should be addressed to Yan Xiong; luyibingli@163.com
}

Received 14 July 2021; Revised 18 August 2021; Accepted 20 August 2021; Published 25 September 2021

Academic Editor: Enas Abdulhay

Copyright (c) 2021 Junqiao Li et al. This is an open access article distributed under the Creative Commons Attribution License, which permits unrestricted use, distribution, and reproduction in any medium, provided the original work is properly cited.

Background. The treatment results of core decompression (CD) and biomechanical support are not always satisfactory in osteonecrosis of the femoral head (ONFH). Stem cell therapy has been incorporated into traditional treatment in order to promote bone regeneration. The efficacy and safety of stem cell therapy combined with CD or biomechanical support on advanced and long-term patients with ONFH were unknown. The aim of this study was to assess whether stem cell combination therapy is superior to single CD or porous tantalum rod implantation treatment in ONFH. Methods. A systematic search of the literature was performed to evaluate all included randomized controlled trials (RCTs) on stem cell combination therapy for patients with ONFH in PubMed, Cochrane Library, Web of Science, and Embase sites. We assessed the quality and risk of bias for the included studies. And the outcomes of Harris hip score (HHS), visual analogue scale (VAS), and adverse events were statistically analyzed. Results. We included 10 randomized controlled trials, containing a total of 498 patients with 719 hips. Stem cell therapy combined with CD versus $\mathrm{CD}$ alone for HHS of ONFH was different $(\mathrm{MD}=8.87,95 \% \mathrm{CI}=[5.53,12.22], P<0.00001)$. The combination of stem cell therapy and CD can effectively improve HHS. Similarly, the VAS of the stem cell combination therapy group also differed compared with the control group $(\mathrm{MD}=-14.07,95 \% \mathrm{CI}=[-18.32,-9.82], P<0.00001)$. The result showed that stem cell combination therapy can relieve the pain of patients with ONFH. There was no significant difference in adverse response outcome events between the combination therapy group and the control group $(\mathrm{RR}=1.57,95 \% \mathrm{CI}=[0.62,3.97], P=0.34)$. Conclusions. Stem cell therapy combined with core decompression is an effective and feasible method with few complications in the clinical treatment of early-stage ONFH. Even in the combination of porous tantalum rod implantation and peripheral blood stem cells, stem cell combination therapy is superior to single biomechanical support treatment. But high-quality, large-sample, multicenter, and long-term follow-up RCTs are still needed to corroborate the efficacy and safety of stem cell combination therapy in ONFH treatment.

\section{Introduction}

Osteonecrosis of the femoral head (ONFH) is a prevalent disease in relatively young patients, usually caused by hip trauma, alcoholism and long-term administration of steroid, which may lead to significant hip pain, articular surface collapse, and eventual osteoarthritis [1]. In clinical treatment, various methods are employed to avert or impede the progression of ONFH. The most common form of therapy is core decompression (CD) that has been universally administered for more than 30 years [2]. However, the treatment results of $\mathrm{CD}$ are not always satisfactory. This method of reconstructing necrotic areas may result in not only inadequate creeping substitution and but also bone remodeling [3]. Therefore, in order to promote bone regeneration, stem cell therapy has been incorporated into traditional CD treatment. When compared with treating ONFH with CD alone, the combined application of stem cell therapy and $\mathrm{CD}$, in early treatment, has superior analgesic and clinical effects, and can more effectively delay the 
progress of femoral head collapse [4]. However, the efficacy and safety of these methods have been controversial and have yet to be proven in patients with advanced and longterm ONFH $[5,6]$.

To investigate the efficacy and safety of stem cell therapy combined with $\mathrm{CD}$ or porous tantalum rod implantation in ONFH patients, especially those with advanced stage and long-term follow-up [7], our research systematically retrieved the latest randomized controlled trials on stem cell combination therapy for ONFH according to the PRISMA guidelines [8]. Furthermore, the positive role and improvement of the new technologies in treatment were discussed, with a view of providing clinicians with scientific evidence for the treatment of ONFH patients.

\section{Materials and Methods}

2.1. Search Strategy. Two researchers independently searched databases from Cochrane Library, Web of Science, PubMed, and Embase. We integrated published randomized controlled studies of cell therapy for femur head necrosis in any language. The search period was from the date of the database establishment to January 2020. A manual supplementary search was performed before submission. The search terms were adjusted according to each database.

Taking PubMed database as an example, the search terms were as follows: ((Femur* Head Necros* [Title/Abstract]) OR (Necros* of Femur* Head [Title/Abstract]) OR ("Femur Head Necrosis" [Mesh])) AND (("Cell-and Tissue-Based Therapy” [Mesh]) OR (Cell-[Title/Abstract] AND TissueBased Therapy [Title/Abstract]) OR (cell therapy [Title/ Abstract]) OR (Cell [Title/Abstract] AND Tissue Based Therapy [Title/Abstract]) OR (Cell Transplantation [Title/ Abstract])) AND ((randomized controlled trial [Publication Type]) OR (controlled clinical trial [Publication Type]) OR (randomized [Title/Abstract]) OR (placebo [Title/Abstract]) OR (randomly [Title/Abstract]) OR (trial [Title]) OR (“Clinical Trials as Topic" [Mesh: noexp])).

2.2. Criteria for Inclusion and Exclusion. In our qualitative and quantitative analysis, only the studies that satisfied the following PICOS criteria were considered: (1) population: patients with stages I to IV of ONFH diagnosed by the Association Research Circulation Osseous classification (ARCO) [9] diagnostic criteria; (2) intervention: cell therapy combined with core decompression or porous tantalum rod implantation; (3) comparator: single core decompression or porous tantalum rod implantation therapy; (4) outcome: HHS, VAS, and adverse events; and (5) study design: randomized controlled trial (RCT).

We excluded studies with the following criteria: (1) republished studies with similar or identical content; (2) dissertation, conference, and review articles; (3) research in animal and basic experimental literature; (4) non-English published research; and (5) nonrandomized controlled trial or other irrelevant studies.
Two researchers followed the criteria for inclusion and exclusion separately. In case of disagreement, a third researcher intervened to resolve it.

2.3. Data Extraction. Two reviewers performed data extraction independently. In case of disputes, differences can be resolved through discussion or third parties until consensus was reached. The extracted information contained baseline information and feature information. The baseline information included: title, study ID, publication country, diagnostic criteria and stages, participants, age, sex ratio, intervention/control group, stem cell source, stem cell counts, the number of hips, and follow-up periods. The feature information included outcome indicators (HHS, VAS), adverse events, effect size, and 95\% confidence interval.

2.4. Quality Assessment. The included studies were independently evaluated by two researchers in accordance with the Cochrane Handbook for Systematic Reviews of Interventions [10]. Cochrane Collaboration Risk of Bias Tool primarily includes selection (taking into account both allocation hiding and random sequence generation), execution (blinding of both subjects and researchers), measurement (research outcomes blind evaluation), and follow-up (outcome data completeness). A total of seven items in six aspects including gender, reporting, and further potential sources of bias were used to assess the bias risk. For each item, a categorization was specified, counting "low-risk bias," "high-risk bias," and "unclear," with this decision being made in accordance with the bias risk assessment criteria.

2.5. Statistical Analysis. Continuous variable values were expressed in mean difference (MD), and a 95\% confidence interval (CI) was calculated for both. Regarding dichotomous variables, we used the risk ratio (RR) and 95\% CI to describe. When $I^{2}>50 \%$, heterogeneity was present within the data; hence, a random-effect model was used. When $I^{2}<50 \%$, there was no heterogeneity present; hence, a fixed effects model was used. The findings of the meta-analysis were shown by the forest plot. We utilized RevMan 5.3 software for statistical analysis. $P<0.05$ was used to evaluate statistical significance. If the number of the included studies was greater than ten, we would use a funnel plot to detect publication bias.

\section{Results}

3.1. Search Results. After searching various databases and a manual search method, we retrieved a total of 123 articles. Mechanical check and manual deduplication were performed by EndNote X9, 47 duplicate articles were deleted, 59 articles were excluded from our analysis after abstract screening, and 7 articles were omitted following a full-text screening, resulting in 10 randomized controlled trials being included in our qualitative and quantitative analysis $[5,6,11-18]$. The flowchart is shown in Figure 1. 


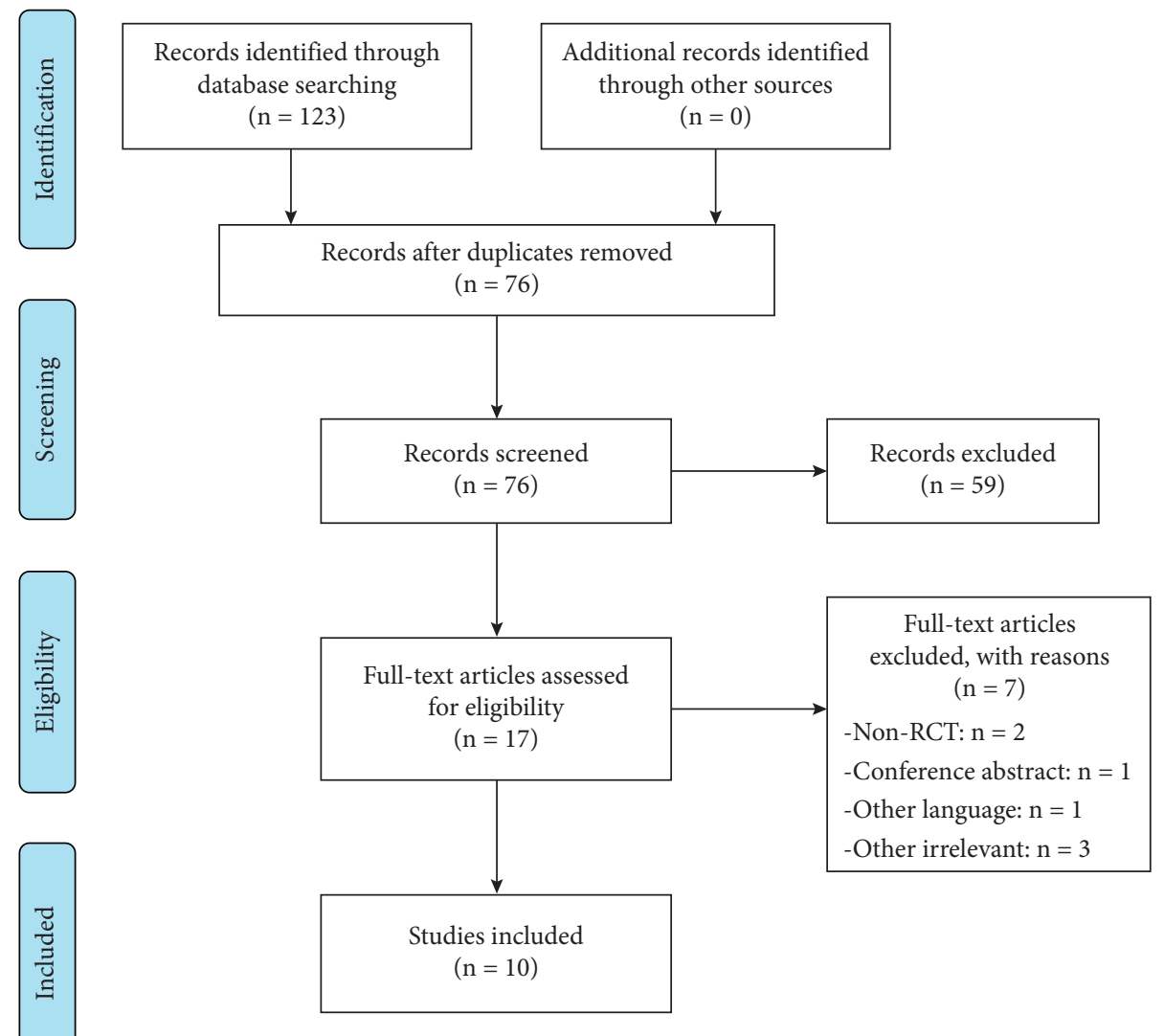

FIGURE 1: Flowchart of literature searching and screening process.

3.2. Study Characteristics. The publication dates of the included RCTs ranged from 2011 to 2018 and belonged to two regions in India $[14,15]$, one in Iran [16], one in Germany [13], three in China $[11,12,17]$, two in Belgium $[5,18]$, and one in France [6]. All the studies used the ARCO stage as the diagnostic criteria. And the disease stage of the included patients with ONFH was mainly seen in the early phase. A total of 498 participants with 719 hips were included in this study. The longest average follow-up time was up to 25 years. See Table 1 for details.

3.3. Quality Assessment. According to the bias risk assessment method recommended by Cochrane Assistance Network, of the 10 studies included, 6 studies [5, 12, 13, 16-18] described the specific random grouping methods, 2 studies $[11,15]$ mentioned random grouping but did not detail the specific method, and the remaining 2 studies $[6,14]$ had a high risk of bias; 4 studies $[6,11,14,17]$ showed the unclear risk of bias for the allocation concealment assessment. As for the blinding of participants and personnel, 4 studies $[6,11,13,14]$ did not report whether double-blind information was used, and one study [17] had a high risk of bias; 4 studies $[6,11,13,14]$ showed the unclear risk of bias for the blinding of outcome assessment. Regarding the incomplete outcome data, one study [13] had an unclear risk of bias, and one study [17] showed a high risk of bias. All studies clearly described the selective reporting and had no other risk bias (Figures 2 and 3).
3.4. Meta-Analysis Results of HHS. Harris hip score was recorded in six studies $[6,12-15,17]$ involving 572 hips. Stem cell therapy combined with core decompression treatment had higher heterogeneity $\left(\mathrm{Chi}^{2}=34.21, I^{2}=85 \%\right.$, $P<0.0001)$ than core decompression alone, and a random effect model was used. The combination treatment group can effectively improve the Harris hip score $(\mathrm{MD}=8.87,95 \%$ $\mathrm{CI}=[5.53,12.22])$, and the difference was statistically significant $(P<0.00001)$. The forest plot is shown in Figure 4 .

3.5. Meta-Analysis Results of VAS. Five studies $[5,6,11,16,18]$ including a total of 397 hips reported visual analogue scales. It was evident that stem cell combination therapy yielded a higher heterogeneity than solely administering core decompression or porous tantalum rod implantation $\left(\mathrm{Chi}^{2}=54.20, I^{2}=93 \%, P<0.00001\right)$, and a random effect model was used. Stem cell combination therapy group can effectively relieve the patients' pain $(\mathrm{MD}=-14.07,95 \% \mathrm{CI}=[-18.32,-9.82])$, with the difference observed between the two groups being statistically significant $(P<0.00001)$. See the forest plot in Figure 5 .

3.6. Meta-Analysis Results of Adverse Events. The perioperative adverse events were reported in five studies $[5,12,13,17,18]$. Of these studies, three studies $[5,12,18]$ reported the adverse response outcome events, including the postoperative pain at the great trochanter and iliac crest, 


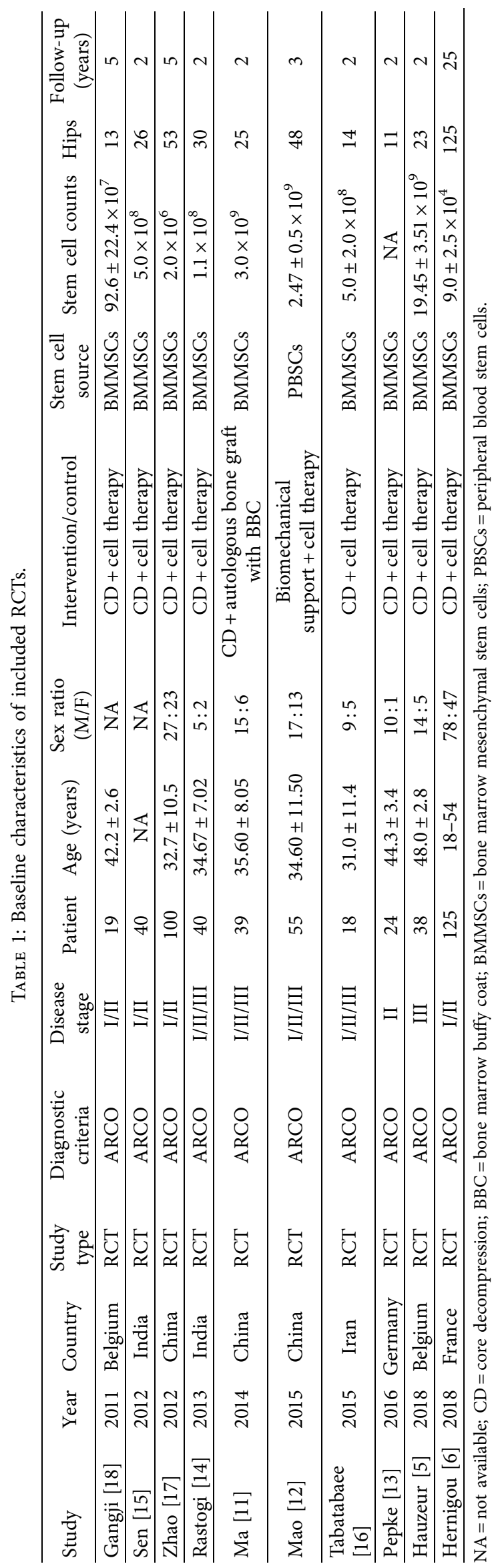




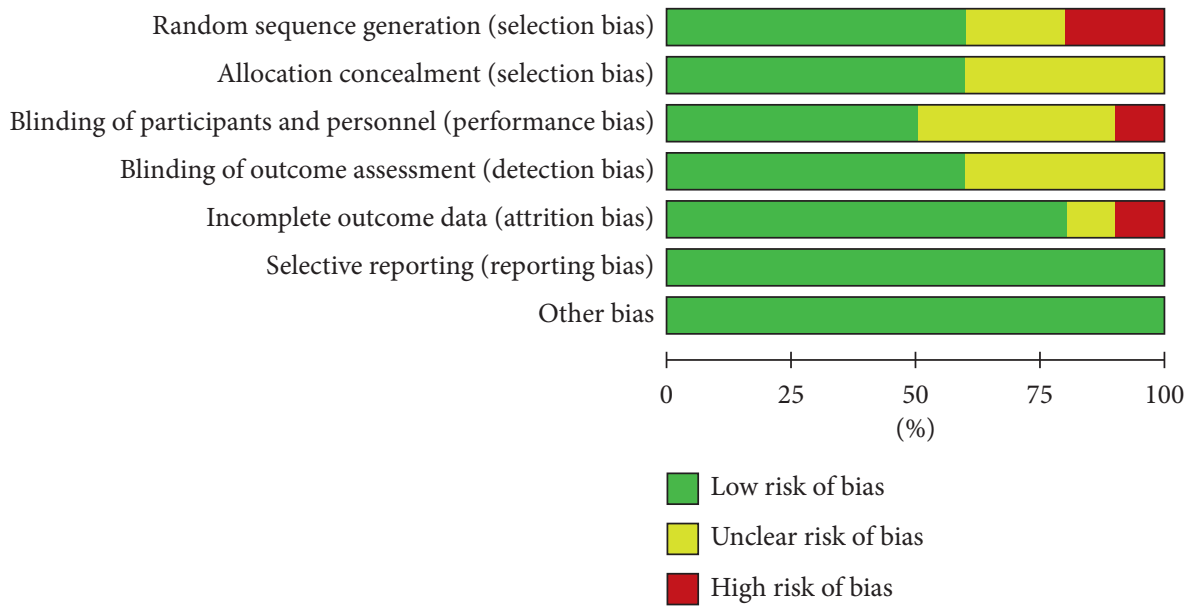

Figure 2: Bias graph risk.

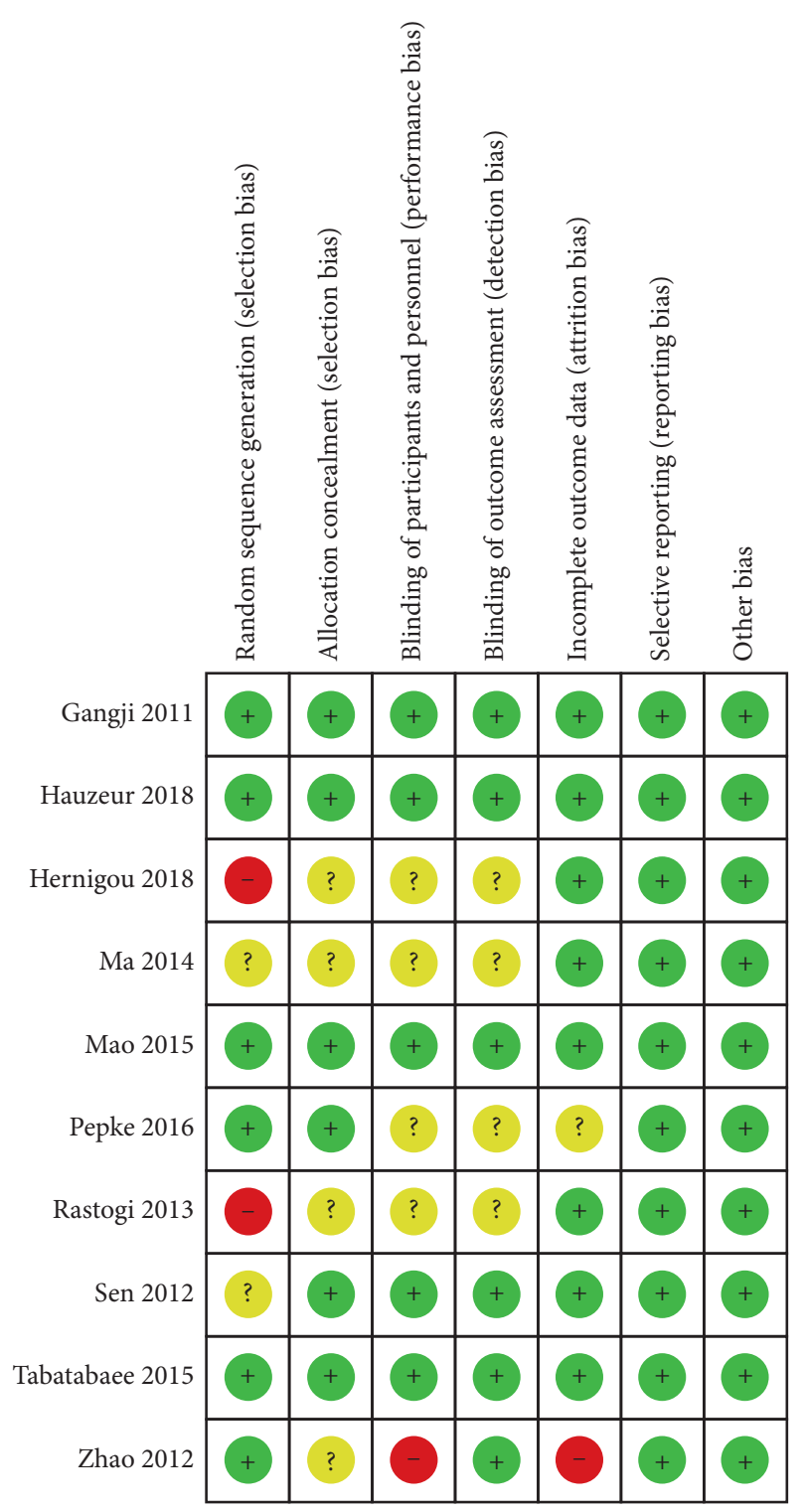

Figure 3: Bias summary risk. hematoma, fever, nausea, infection, and porous tantalum rod displaced. Two studies $[13,17]$ reported that no adverse events were observed in both groups during the study period. The analysis showed that there was no significant difference in adverse events between the stem cell combination therapy group and the control group $(\mathrm{RR}=1.57,95 \%$ $\mathrm{CI}=[0.62,3.97], P=0.34)$. The forest plot is shown in Figure 6.

3.7. Publication Bias. As no more than ten published studies were included, it was not possible to assess publication bias for the time being.

\section{Discussion}

4.1. Main Findings. Our findings demonstrate that in earlystage $\mathrm{ONFH}$, stem cell therapy combined with core decompression is far more effective than core decompression alone. And the combination therapy has good safety with few complications. Moreover, even in the combination of porous tantalum rod implantation and peripheral blood stem cells, stem cell combination therapy is superior to single biomechanical support treatment.

4.2. Effectiveness of Stem Cell Combination Therapy. Stem cell combination therapy can relieve the symptoms of hip pain, improve patients' HHS, halt disease progression, and result in a reduction in the incidence of total hip replacement for the early-stage ONHF patients. This was confirmed in three independent systematic reviews conducted by Papakostidis et al. [19], Piuzzi et al. [20], and Wang et al. [4], respectively. However, in advanced patients, the evolution of necrosis was not significantly improved by stem cell therapy after core decompression [5]. Furthermore, the results of a network meta-analysis study by Yoon et al. [21] questioned the natural course of ONFH by CD treatment. The findings of this study suggested that the small lesions will not collapse, even without treatment being administered. This conclusion 


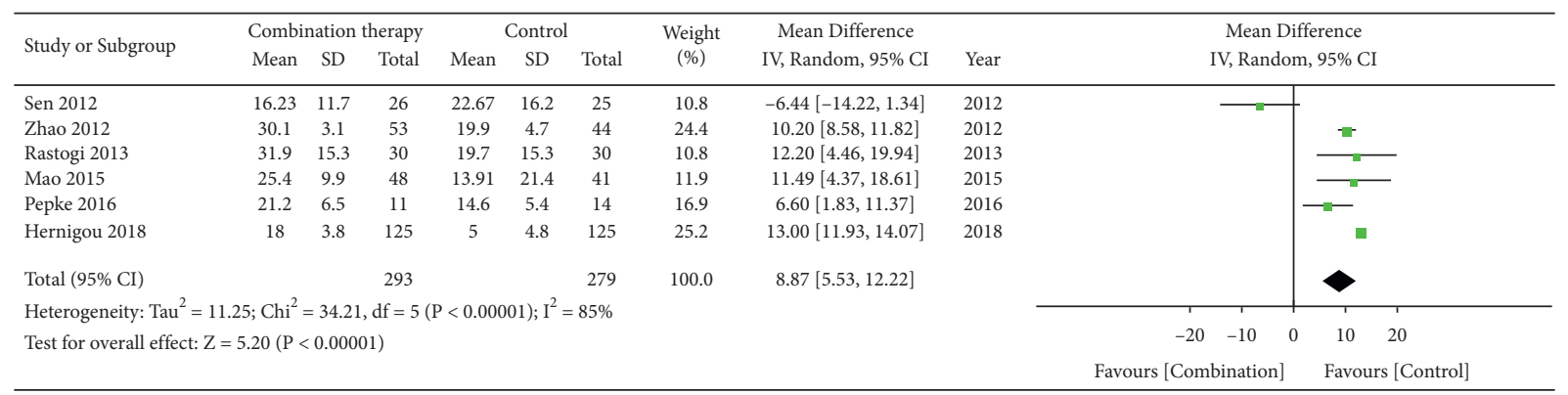

FIGURE 4: Forest plot of HHS.

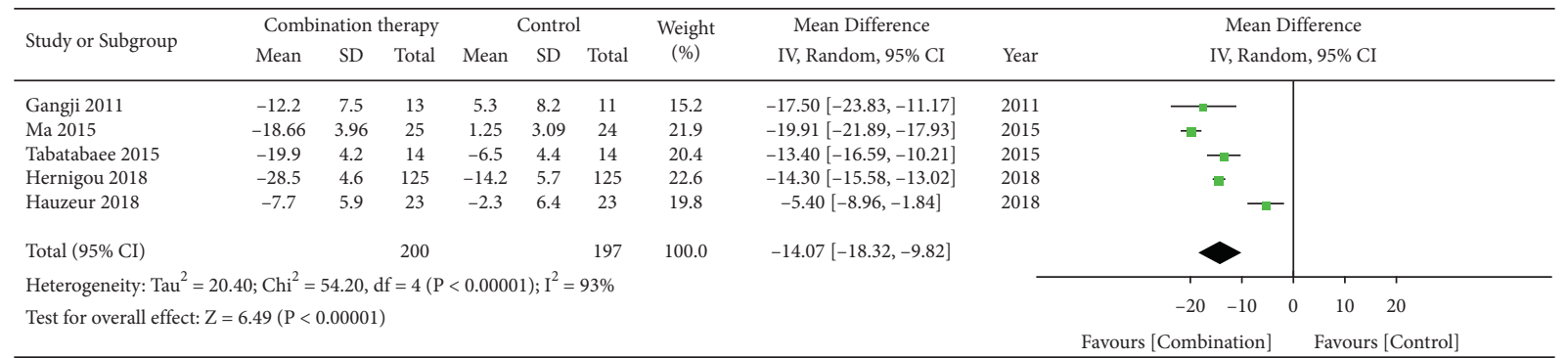

Figure 5: Forest plot of VAS.

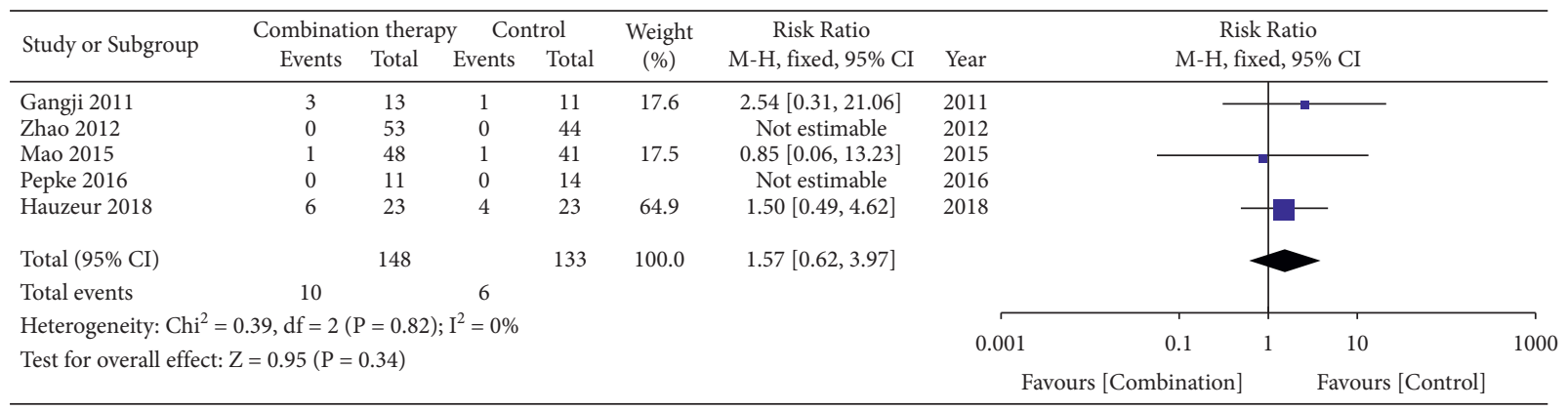

Figure 6: Forest plot of adverse events.

took into account that the extent of the decaying component was the primary factor of the necrotic femoral head fracture's collapse. However, even though the progression of osteonecrosis may result from numerous factors [6], an average follow-up time of 25-year prospective randomized study by Hernigou et al. [6] established that bone marrow cell implantation of necrotic lesions may potentially offer an effective treatment for early femoral head necrosis and impede the evolution of ailment, lessen the incidence of femoral head collapse, and elude the arthroplasty even at long-term follow-up.

4.3. Safety of Stem Cell Combination Therapy. The probability of adverse complications was extremely low, especially in the aspects of infection, excessive new bone formation, tumor induction, and local complications on the surviving side [13]. Stem cell therapy was safe for the treatment of ONFH. Even after 3-10 years of follow-up, there were no complications related to malignant tumors, bone overgrowth, core tract fractures, perforation of femoral head, deep vein thrombosis, infection, and so forth [7]. Ma et al. [11] conducted a prospective, double-blinded, randomized, controlled investigation. In order to reduce the failure rate, they improved the technology and used autologous bone grafts obtained by ring drilling with bone marrow buffy coat. Hernigou et al. [6] corroborated these findings and noted that computer navigation had the potential to be safely implemented in a basic procedure for the injection of stem cells. They all improved the overall safety of stem cell therapy and reduced the probability of complications. Additionally, Ciapetti et al. [22] demonstrated that, compared with bone marrow mesenchymal stem cells under normal conditions, the proliferation and colonization capacity of these stem cells were significantly enhanced in a hypoxic environment.

4.4. Strengths and Limitations. This systematic review was ultimately included in ten studies. All the studies were RCTs of stem cell combination therapy for ONFH, which would provide us with strong evidence for the efficacy and safety of this method in ONFH treatment. Owing to the small 
number of included studies, we could not make funnel charts to determine publication bias. The heterogeneity in the outcome indicators was large, which may result from certain differences in the included studies in aspects of stem cell concentration, treatment time, and the quality of the literature, leading to greater heterogeneity in each clinical study, and some outcome indicators were not stable.

The findings of this meta-analysis still had certain limitations: (1) the overall quality of the scope of included literature was not high, the sample size was small, and the short follow-up time in some studies may also lead to potential bias; (2) as no more than ten studies were included, publication bias cannot be assessed; (3) publication in English may result in language or regional bias; and (4) there was some heterogeneity in outcome indicators. Highquality, large-sample, multicenter, and long-term follow-up randomized controlled trials are still warranted to corroborate the differences in the efficacy and safety of stem cell combination therapy in ONFH.

\section{Conclusion}

Stem cell therapy combined with core decompression is an effective and feasible method with few complications in the clinical treatment of early-stage ONFH. Even in the combination of porous tantalum rod implantation and peripheral blood stem cells, stem cell combination therapy is superior to single biomechanical support treatment. But high-quality, large-sample, multicenter, and long-term follow-up RCTs are still needed to corroborate the efficacy and safety of stem cell combination therapy in ONFH treatment.

\section{Data Availability}

All data included in this study are available upon request by contact with the corresponding author.

\section{Conflicts of Interest}

All authors declare no conflicts of interest with this work.

\section{Authors' Contributions}

All authors read the manuscript and agreed to publish it.

\section{References}

[1] E. Larson, L. C. Jones, S. B. Goodman, K.-H. Koo, and Q. Cui, "Early-stage osteonecrosis of the femoral head: where are we and where are we going in year 2018?" International Orthopaedics, vol. 42, no. 7, pp. 1723-1728, 2018.

[2] I. Learmonth, S. Maloon, and G. Dall, "Core decompression for early atraumatic osteonecrosis of the femoral head," Journal of Bone \& Joint Surgery, British Volume, vol. 72-B, no. 3, pp. 387-390, 1990.

[3] K. Koo, R. Kim, G. Ko, H. Song, S. Jeong, and S. Cho, "Preventing collapse in early osteonecrosis of the femoral head. a randomised clinical trial of core decompression," Journal of Bone \& Joint Surgery, British Volume, vol. 77-B, no. 6, pp. 870-874, 1995.
[4] Z. Wang, Q.-M. Sun, F.-Q. Zhang, Q.-L. Zhang, L.-G. Wang, and W.-J. Wang, "Core decompression combined with autologous bone marrow stem cells versus core decompression alone for patients with osteonecrosis of the femoral head: a meta-analysis," International Journal of Surgery, vol. 69, pp. 23-31, 2019.

[5] J.-P. Hauzeur, V. De Maertelaer, E. Baudoux, M. Malaise, Y. Beguin, and V. Gangji, "Inefficacy of autologous bone marrow concentrate in stage three osteonecrosis: a randomized controlled double-blind trial," International Orthopaedics, vol. 42, no. 7, pp. 1429-1435, 2018.

[6] P. Hernigou, A. Dubory, Y. Homma et al., "Cell therapy versus simultaneous contralateral decompression in symptomatic corticosteroid osteonecrosis: a thirty year follow-up prospective randomized study of one hundred and twenty five adult patients," International Orthopaedics, vol. 42, no. 7, pp. 1639-1649, 2018.

[7] J. S. Kang, Y. J. Suh, K. H. Moon et al., "Clinical efficiency of bone marrow mesenchymal stem cell implantation for osteonecrosis of the femoral head: a matched pair control study with simple core decompression," Stem Cell Research \& Therapy, vol. 9, no. 1, 2018.

[8] D. Moher, A. Liberati, J. Tetzlaff, D. G. Altman, and P. Group, "Preferred reporting items for systematic reviews and metaanalyses: the PRISMA statement," Annals of Internal Medicine, vol. 151, no. 4, pp. 264-269, 2009.

[9] J. W. M. Gardeniers, "ARCO (association research circulation osseous) committee on terminology and classification," ARC News, vol. 5, pp. 79-82, 1993.

[10] J. J. Deeks, J. Higgins, D. G. Altman, and S. Green, Cochrane Handbook for Systematic Reviews of Interventions Version 5.1. 0 (Updated March 2011), The Cochrane Collaboration, London, UK, 2011, http://www.cochrane-handbook.org.

[11] Y. Ma, T. Wang, J. Liao et al., "Efficacy of autologous bone marrow buffy coat grafting combined with core decompression in patients with avascular necrosis of femoral head: a prospective, double-blinded, randomized, controlled study," Stem Cell Research \& Therapy, vol. 5, no. 5, p. 115, 2014.

[12] Q. Mao, W. Wang, T. Xu et al., "Combination treatment of biomechanical support and targeted intra-arterial infusion of peripheral blood stem cells mobilized by granulocyte-colony stimulating factor for the osteonecrosis of the femoral head: a randomized controlled clinical trial," Journal of Bone and Mineral Research, vol. 30, no. 4, pp. 647-656, 2015.

[13] W. Pepke, P. Kasten, N. A. Beckmann, P. Janicki, and M. Egermann, "Core decompression and autologous bone marrow concentrate for treatment of femoral head osteonecrosis: a randomized prospective study," Orthopedic Reviews, vol. 8, pp. 6162-6169, 2016.

[14] S. Rastogi, S. R. Sankineani, H. L. Nag et al., "Intralesional autologous mesenchymal stem cells in management of osteonecrosis of femur: a preliminary study," Musculoskeletal surgery, vol. 97, no. 3, pp. 223-228, 2013.

[15] R. K. Sen, S. K. Tripathy, S. Aggarwal, N. Marwaha, R. R. Sharma, and N. Khandelwal, "Early results of core decompression and autologous bone marrow mononuclear cells instillation in femoral head osteonecrosis," The Journal of Arthroplasty, vol. 27, no. 5, pp. 679-686, 2012.

[16] R. M. Tabatabaee, S. Saberi, J. Parvizi, S. M. J. Mortazavi, and M. Farzan, "Combining concentrated autologous bone marrow stem cells injection with core decompression improves outcome for patients with early-stage osteonecrosis of the femoral head: a comparative study," The Journal of Arthroplasty, vol. 30, no. 9, pp. 11-15, 2015. 
[17] D. Zhao, D. Cui, B. Wang et al., "Treatment of early stage osteonecrosis of the femoral head with autologous implantation of bone marrow-derived and cultured mesenchymal stem cells," Bone, vol. 50, no. 1, pp. 325-330, 2012.

[18] V. Gangji, V. De Maertelaer, and J.-P. Hauzeur, “Autologous bone marrow cell implantation in the treatment of nontraumatic osteonecrosis of the femoral head: five year followup of a prospective controlled study," Bone, vol. 49, no. 5, pp. 1005-1009, 2011.

[19] C. Papakostidis, T. H. Tosounidis, E. Jones, and P. V. Giannoudis, "The role of "cell therapy" in osteonecrosis of the femoral head," Acta Orthopaedica, vol. 87, no. 1, pp. 72-78, 2016.

[20] N. S. Piuzzi, J. Chahla, J. B. Schrock et al., "Evidence for the use of cell-based therapy for the treatment of osteonecrosis of the femoral head: a systematic review of the literature," The Journal of Arthroplasty, vol. 32, no. 5, pp. 1698-1708, 2017.

[21] B.-H. Yoon, Y.-K. Lee, K.-C. Kim, Y.-C. Ha, and K.-H. Koo, "No differences in the efficacy among various core decompression modalities and non-operative treatment: a network meta-analysis," International Orthopaedics, vol. 42, no. 12, pp. 2737-2743, 2018.

[22] G. Ciapetti, D. Granchi, C. Fotia et al., "Effects of hypoxia on osteogenic differentiation of mesenchymal stromal cells used as a cell therapy for avascular necrosis of the femoral head," Cytotherapy, vol. 18, no. 9, pp. 1087-1099, 2016. 